\title{
A GLOBAL FORMALISM INCORPORATING CONSTANTS OF MOTION AND OTHER CONSTRAINTS IN THE CLASSICAL DESCRIPTION OF CHEMICAL RATE PROCESSES
}

\author{
Erık K. GRIMMELMANN ${ }^{\ddagger}$ and Lawrence L. LOHR Jr \\ Department of Chemistry. Unuversity of Michigan Ann Arbor, Michigan 48109, USA
}

Recelved 15 August 1979; in final form 30 October 1979

\begin{abstract}
A phase space cell method for providing a global description of the motions of a physical system ts outlined. The key feature 15 a coarse-graining approximation to the phase-space probabilsty distribution. Constants of motion and other constraints are incorporated to reduce the dimensionality of computations Time-independent and time-dependent methods of solution are discussed.
\end{abstract}

\section{Introduction}

The calculation of average dynamical properties of chemical systems from the interactions of individual atoms and molecules is of ten practically very difficult although conceptually relatively simple Given a potential energy hypersurface for the atoms comprising a system, it is easy (although perhaps time consuming) to integrate the classical equations of motion to obtain representatuve phase trajectories for the system [1] To characterize accurately the dynamical properties of the system, however, may require averaging over so many initial degrees of freedom that the number of trajectories required is so large that the calculation becomes unfeasible. In the present work we outline a method in which the motions of a system are followed simultaneously for appropriate distributions of initial conditions, rather than for individual initial conditions as is done in traditional trajectory calculations [2] . Our approach is thus global, rather than local In addition, our method, which we designate as the "phase space cell method" (PSCM), explicitly utuizes the constraints on a system to reduce the dimensionality of the calculation.

In the present work we focus our attention on only part of the phase space associated with the system of interest. In this respect this work is somewhat sim-

\footnotetext{
₹ Present address Bell Laboratories, Murray Hıll, Neu Jersey 07974, USA
}

Ilar to the approaches of others in which only part of the complete phase space is considered and in which master equation methods are used [3-6]. The present work is also somewhat similar in spirit to the wave packet approach of Heller [7].

\section{General considerations}

We consider a system with $3 N$ degrees of freedom and describe the motion of its $3 N-6$ internal degrees of freedom using the generalized coordinates $q$ and their conjugate momenta $p$. We assume that the motion of the system can be described classically and that the potential energy $V(q)$ and its partial derivatives $\partial V(q) / \partial q_{2}$ are known. In addition, we restrict our attention to a fixed value of the total energy $E$. The standard approach to characterizing the macroscopic dynamic behavior of such a system is to consider representative trajectories of the system [1]. One numerically integrates Hamilton's equations of motion for many "initial" conditions chosen from the appropriate microcanonical ensemble and obtains time dependent distributions of observable quantities. For example, different regions of phase space may ba identified with different product states or species so that transition rates or branching ratios can be calculated.

While examining "representative" individual trajectories is occasionally useful in identifying "typical" patterns of motion, generally one is more interested in 
average quantities In fact, it is often only average quantities which are physıcally observable Calculatıng classical trajectories is certainly straightforward. but it also provides much more information than is generally needed. Furthermore, constraints upon the system are rarely utilized in integrating the equations of motion. These constrainis may be mherent in the mechanics. such as the conservation of energy and momentum. or they may be constraints which are inposed upon a system This second type of constraint includes, for example, the restriction that the atoms comprising a system be collinear or coplanar. It also includes the restriction that the initial energy in a particular vibrational or rotational mode be fixed at its corresponding quantum mechanical value, as is done in quasiclassical trajectory calculations

We next present a method in which the constraints on a system are used to iaduce the diniensionality of the calculation and in which the motions of a system are followed sinultaneously for approprate dist ributions of initial conditions. rather than separately for individual initial conditions

\section{General method}

We now consider the general features of the phase space cell method and, in the subsequent sections, present two alternate forms in which it may be used [2]. We first seek to choose a "good" set of generalized coordinates $q$, ones which convenient ly describe the system of interest We choose these coordinates with several criteria in mind. First , they should naturally portray the motion of the system (e $g$ as natural collision coordinates do for reactive scattering systemis). Second, they should separate the internal degrees of freedom from the uninteresting translation of and rotation about the center of mass And third, they should lend themselves to the explicit inclusion in the calculation of the other constraints upon the system Of course, no coordinates will be ideal in all three of these respects, so some compromises will be necessary in choosing the best coordinates for a particular system. The phase space of the system, which we designate as $\Gamma^{0}$, consists of the coordinates $q$ and their conjugate momenta $p$. The constant energy hypersurfaces of $\Gamma^{0}$ may be either finite or infinite, depending on the nature of the system. They are finite for bound systems and infinite if the system can separate into at least two parts which can recede infinitely far from each other in configuration space. If the constant energy liypersurfaces of $\Gamma^{0}$ are finite, we consider all of $\Gamma^{0}$, whlle if they are infinite, we consider only that part of $\Gamma^{0}$ for which all particles or groups of particles are non-negligibly interacting with the rest of the system. We designate as $\Gamma$ that portion of $\Gamma^{0}$ upon which we focus attention.

We now consider a microcanonical ensemble of systems representing the physical system of interest. In application to a reaction $\mathrm{A}+\mathrm{BC} \rightarrow \mathrm{AB}+\mathrm{C}$, say, a "-system" will consist of a single molecular unit $A B C$, so that the dimensionality of $\Gamma^{0}$ is twice the number of position coordinates used to describe ABC From this ensemble we choose a portion of the representative systems on which to focus our at tention. We postpone for the moment a full discussion of which representative systems we choose. The phase density $\rho_{0}(q \cdot p . t)$ is then the probability density at the pliase point $(\boldsymbol{q}, \boldsymbol{p})$ at time $t$. such that $\rho_{0} \mathrm{~d} \tau=\rho_{0} \mathrm{~d} q \mathrm{~d} p$ is the fraction of system points to be found in the volume $\mathrm{d} \tau$ about the point $(\boldsymbol{q} \cdot \boldsymbol{p})$ at time $t$ Of course, $\rho_{0}$ can be non-zero only on the hypersurface(s) of $\Gamma$ which satisfy the constraints on the system For convenience of notation, let $\rho(q, p, t)$ be a function whose value is equal to the value of $\rho_{0}(q, p, t)$ for all points $(q, p)$ on the hypersurface(s) of $\Gamma$ which sat isfies the constraints on the system and whose value is arbitrary elsewhere

We now consider the phase space $\Gamma$ and divide it into "cells" These cells need not be all the same size or shape; the only requirement on them is that each phase point in $\Gamma$ be in one and only one cell We label the cells by the index $i$, letting $c_{z}$ designate the region comprising the $t$ th cell Further, we designate the probability that a representative system be within cell $t$ at time $t$ by $P_{l}(t)$, which is given by

$P_{l}(t)=\int_{c_{l}} \rho(\tau, t)\left[\prod_{I=1}^{n} \delta\left(c_{J}(\tau)\right)\right] \mathrm{d} \tau$

Here $\tau \equiv(q, p)$ and the product is over the various constraints on the system Each of the $\boldsymbol{n}$ constraints is represented by a Dirac delta function For example, the constraint that the total energy is fixed is represented by $\delta(E-E(\tau))$. where $E$ is the total energy of the system and $E(\tau)$ is the total energy at the point $\tau$ in phase space The quantity $P_{l}(t)$ is just the coarse- 
grained probability at time $t$ If the division of phase space into cells is fine enough, this course-grained probabilıty can be used to describe adequately the dynamical behavior of the system.

Choosing a tıme interval $\Delta t$, we have $\Delta P_{l}(t, \Delta t)=$ $P_{I}(t+\Delta t)-P_{I}(t)$ A system whose representative point is within cell $l$ at time $t$ may have its representative point within a different cell, say cell $j$, at time $t+\Delta t$. We designate as $c_{i j}(\Delta t)$ the portion of cell $i$ whose points are connected by phase trajectories of duration $\Delta t$ to points in cell $j$. Thus, all systems whose representative points are within $c_{l j}(\Delta t)$ at any time $t$ have their representative points within cell $j$ at time $t+\Delta t$. The regions $c_{l j}(\Delta t)$ depend upon the interval $\Delta t$ chosen but not upon the absolute time $t$; they are most easily determined by calculating trajectones of duration $\Delta t$ beginning at a number of points withun cell $l$ and seeing which of them end in cell $l$ If the constant energy hypersurfaces of the full phase space $\Gamma^{0}$ are infinite, the regions of $\Gamma^{0}$ which have not been divided into cells are also included in the indexing scheme We designate by $c_{i *}(\Delta t)$ the region of cell $i$ from which density flows out of the subspace $\Gamma$ during the interval $\Delta t$.

We can now write an expression for $\Delta P_{l}(t, \Delta t)$,

$$
\begin{gathered}
\Delta P_{l}(t, \Delta t)=-\sum_{t \neq l} F_{l}(t, \Delta t)-D_{l}(t, \Delta t) \\
+\sum_{t \neq l} F_{l l}(t, \Delta t)+E_{l}(t, \Delta t),
\end{gathered}
$$

where

$$
F_{m n}(t, \Delta t)=\int_{c_{m n}(\Delta t)} \rho(\tau, t)\left[\prod_{l=1}^{n} \delta\left(c_{l}(\tau)\right)\right] \mathrm{d} \tau
$$

and

$$
D_{m}(t, \Delta t)=\int_{c_{m *}(\Delta t)} \rho(\tau, t)\left[\prod_{J=1}^{n} \delta\left(c_{J}(\tau)\right)\right] \mathrm{d} \tau .
$$

The first sum on the $r \mathrm{~h} s$ of eq. (2) is the probability flowng out of cell $l$ and into other cells, while the second term is the probability flowing from cell $l$ into that part of phase space which is not divided into cells (a "sink"). The third term is the probability flowing into cell $l$ from other cells in the region $\Gamma$, while the final term on the r.h s. of eq. (2) is the probability flowing into cell $\boldsymbol{t}$ from that portion of phase space which is not divided into cells (a "source"). If the constant energy hypersurfaces of $\Gamma^{0}$ are finite, then all of $\Gamma^{0}$ is divided into cells and the $D_{t}$ and $E_{t}$ are all zero.

We obtain an expression for $\dot{P}_{i}(t)=\mathrm{d} \boldsymbol{P}_{i}(t) / \mathrm{d} t$ by approximating it by $\Delta P_{i}(t, \Delta t) / \Delta t$,

$$
\begin{gathered}
\dot{P}_{i}(t)=-\sum_{j \neq l} F_{i j}\left(t, \Delta t_{l}\right) / \Delta t_{i}-D_{i}\left(t, \Delta t_{i}\right) / \Delta t_{i} \\
+\sum_{j=1} F_{j l}\left(t, \Delta t_{J}\right) / \Delta t_{j}+\dot{E}_{i}(t) .
\end{gathered}
$$

The source term $\dot{E}_{z}(t)$ is not written as a ratio of finite differences as it can usually be explicitly specified. We have labeled the $\Delta t$ 's by the index of the cell from whuch the probability is flowing to indicate that a different time interval may be used for calculating the probability flowing from each cell. The use of different $\Delta t$ 's can improve the efficiency and accuracy of the calculation since the velocities of representative points can vary widely from one region of $\Gamma$ to another, and a $\Delta t$ which would be suitable in one region might not be suitable in another.

We wish to obtain the $P_{l}(t)$ and from these coarsegrained probabilities determine the dynamical behavior of the system. In what follows we make the assumption that within each cell the phase density is independent of location withun the cell. Thus

$\rho(\tau, t)=P_{2}(t) / A_{2}$,

where $A_{z}$ is the portion of the hypervolume of the $i$ th cell satisfying the constraints on the problem and is given by

$A_{l}=\int_{c_{l}}\left[\prod_{l=1}^{n} \rho\left(c_{j}(\tau)\right)\right] \mathrm{d} \tau$.

This assumption is not the only one which could be made, inştead we could assume, for example, that $\rho(\tau, t)$ were given by a polynomial fit to the phase density at the center of cell $i$ and the centers of a limited number of neighboring cells. In the present work, however, we stick with the assumption that $\rho(\tau, t)$ is independent of location within each cell. Using this assumption, eq. (4) may be rewritten as

$\dot{P}_{I}(t)=\sum_{I} G_{t J} P_{I}(t)+\dot{E}_{t}(t)$,

where 
$G_{1 l}=-\frac{1}{A_{l} \Delta t_{l}}\left[\sum_{j=l} B_{l j}+B_{l *}\right]$

and

$G_{l j}=-\frac{1}{A_{j} \Delta t}\left[\sum_{f=1} B_{j l}\right]$

Eq (7) represents a set of coupled first-order Inear differential equations (a "master equation") for the $P_{l}(t)$ The vector elements $A_{l}$ and $A_{l}$ in eqs (8a) and (8b) are given by eq (6). wlile the array elements $B_{l l}$ are given by a simular expression with $c_{t}$ replaced by $c_{l j}(\Delta t)$, the $B_{l j}$ represent the combined portions of the liy'pervolumes of cells $\boldsymbol{l}$ and $\boldsymbol{J}$ that are connected by phase trajectories of duration $\Delta t$ from cell $l$ to cell J The symbol * as betore denotes the "sink" region

There are two possible approaches to obtaining the $P_{\boldsymbol{l}}(t)$ tron eq $(7)$ The first approach is the tine-independent case for which we obtan a set of steady-state $P_{1}$. The second approach is the time-dependent case for whicl we obtain the time evolution of a "probability packet " through phase space.

\section{Time-independent solution}

The time-independent approach is suitable for cases where the constant energy hy'persurfaces of the full phase space $\Gamma^{0}$ are infinite. Typically, in these cases. several species approach each other from infinite separation and begin interacting non-negligibly only when they are within a certain distance of each other We assume, then, that there is a constant probability flux into the subspace $\Gamma$ from the region of $\Gamma^{0}$ which is not also contained in $\Gamma$. Under these conditions, a time-independent (1 e , steady-state) solut ion of (4) will exist provided all probability flowing into $\Gamma$ eventually leaves $\Gamma$ Finding a steady-state solution is difficult, however. if all trajectories entering $\Gamma$ do not leave $\Gamma$ within a reasonable length of time. In cases where such long lived complexes exust, the tme-dependent approach, which will be out lined in the following section, would have to be applied.

If a steady-state solution exists, it can be obtained by setting $\dot{P}_{t}(t)$ to zero in eq (7), which leads to

$\sum_{J} G_{l j} P_{l}+\dot{E}_{l}=0$
This is simply a set of simultaneous linear equations which is readily solved using an iterative technique [8]. Once the steady-state $P_{l}$ are obtained, the dynamical properties of the system are easy to calculate. If, for evample, the probability flowing out of $\Gamma$ ends up in two distinct regions of $\Gamma^{0}$ which correspond to distinguishable product states, then the branching ratio for the react ion is simply the ratio of the fluxed flowing into the two regions

\section{Time-dependent solution}

We now consider the time-dependent approach for the phase space cell method In contrast to the timeindependent approach which is suitable for certain systems only, the tme-dependent approach is suitable for all systems For the time-dependent solution, the system is started at time zero with a specified phase distribution. Eacli particular choice of initial conditions [ 1 e , each set of $P(0)$ ] evolves differently' with time The particular $P_{l}(0)$ chosen may consist of the initial probabihty being all within one cell, or it may consist of some distribution of probability among a number of cells Unlike in the time-independent case, in the t ime-dependent case there is no probability flowing into cells from the region of $\Gamma^{0}$ which is not divided into cells, that is $\dot{E}_{l}(t)$ is zero. Since $\dot{E}_{l}(t)$ is zero eq (7) may be written as

$\dot{P}_{l}(t)=\sum_{l} G_{l j} P_{l}(t)$

Eq. (10) is a set of homogeneous, first-order linear differential equations with constant coefficients which are readily solved using standard techniques. Once the $P_{I}(t)$ are known, the dynamical properties of the system can be readily determined.

\section{Computational procedure}

In order to clarify the computational procedure we have suggested, we now present a sequence of steps which could be followed to apply the PSCM. This sequence represents only one of many possible ways in which this method could be implemented.

(1) Choose coordinates and divide the phase space of the system into cells. 
(2) Specify the constraints on the system and put them in terms of a set of delta functions [the $\delta\left(c_{I}(\tau)\right)$ in eq. (1)].

(3) Follow the motion of the center of each cell for a specified tıme or until it moves a specified distance Then, assuming that each point within a cell moves parallel to the path of motion of the center of the cell, determine which portions of each cell move to each adjacent cell and thus obtain the $c_{l_{j}}(\Delta t)$ and $c_{l *}(\Delta t)$.

(4) Calculate the integrals of the type given in eq. (1) to determine the various $A_{l}, B_{l}$, and $B_{l *}$. If necessary these integrals may be computed numerically.

(5) Use eqs. (8a) and (8b) to obtain the $G_{l y}$.

(6) Choose whether to find the time-independent or the time-dependent solution.

(7) Specify the initual conditions. These are the $\dot{E}_{z}$ for the time-independent case and the $P_{l}(0)$ for the time-dependent case.

(8) Solve eq. (9) or (10) to obtain the steady-state $P_{l}$ or the tume dependent $P_{l}(t)$.

(9) Use the $P_{1}$ to obtain the rate information desired.

\section{Further comments}

We mentioned previously that in using the phase space cell method we consider a microcanonical ensemble of systems representing the physical system of interest and from this ensemble choose a portion of the representative systems upon which to form our attention We now discuss in further detail this choice of representative systems. Choosing different representative systems corresponds to considering the initial conditions of the physical system of interest For the tıme-independent solution of the problem, the initial conditions enter through the $\dot{E}_{l}$, while for the time-dependent solution they enter through the $P_{l}(0)$

It is necessary, of course, for the initial conditions to satisfy the constraints imposed upon the system However, the initial conditions may be restricted beyond what is required for the constraints on the system. Thus, for example, the pseudo-quantization of a particular degree of freedom could be included in the initial conditions of a problem while not being included as an explicit constraint upon the system.

Throughout this work we have discussed the "flow" of probability through phase space, we now compare thus flow to the flow of a physical fluid and considee the applicability of some of the methods of fluid dynamics to the phase space cell method. First, we note from Liouville's theorein that the true phase density is incompressible [9]. If we were to relax the assumpton of eq. (5) that at each time the phase density is independent of location withun a cell in such a way that the density is continuous at the cell boundaries, then the techniques of fluid mechanics for ideal incompressible fluids [10] could be used directly in solving eq. (4). While the dynamics of ideal incompressible fluids and the coarse-grained probability of the phase space cell model are largely equivalent, this equivalence does not exist in the absence of coarsegraining. The motion of a small volume of fluid depends, in general, upon the motion of neighboring volumes of fluids; that is, the streamlines of a system are a global and not a local property. Simularly, by coarsegraining, the motion of a small element of probability also depends upon the motion of neighboring elements. The evolution of individual phase trajectories, however, is independent of nearby trajectortes.

\section{Summary}

In this work we have outlıned a method in which the motions of a physical system are followed simultaneously for appropriate distributions of initial conditions. This is a global rather than a local approach to the problem. In addition, the constraints on the system are used explicitly to reduce the dimensionality of the calculation. By providing a global "picture" of a chemical reaction, this method can potentially provide a more intuitive description of chemical processes than can be provided by traditional trajectory methods.

The consequences of employing a coarse-grained ensemble density has been discussed by Liboff, followIng an outline due to Gibbs. Liboff [1 1 ] points out that to know a coarse-granned density $\pi_{i}(t)$, equivalent in our notation to $P_{t}(t) / A_{l}$, is to know considerably less than it is to know the dynamical function $D$ (our $\left.\rho_{0}\right)$. The irreversibility of macroscopic physics follows from the forming of macroscopic averaged (coarsegrained) variables.

In conclusion it should be noted that we have already carned out a numerical study $[2,12]$ of the dynamics of the collunear $\mathrm{H}+\mathrm{H}_{2}$ exchange reaction employing the time-independent method of this paper. 


\section{References}

[1] D L. Bunker, Methods Comput. Phys 10 (1971) 287, R N. Porter, Ann. Rev. Phys Chem 25 (1974) 317. R.N.Poiter and L M. Rafi, Dynamics of molecular collisions, Part B, ed W H. Miller (Plenum Press, New York, 1976)

[2] E K. Grummelmann, Ph.D Thesis, The University of Michigan (1978)

[3] S.D. Augustin and $H$ Rabitz. J Chem Phys 64 (1976) $1223 ; 66$ (1977) 269;67 (1977) 64

[4] G C Schatz, J. Chem Phys 66 (1977) 5220, Chem Phys. 24 (1977) 263; Mol. Phys 35 (1978) 477

[5] G C Schatz, F.J. McLafierty and J Ross, J. Chem Phys 66 (1977) 3609 .
D J Zvıjac, S Mukamel and J. Ross, J. Chem. Phys 67 (1977) 2007.

[6] B C Eu, Chem Phys Letters 47 (1977) 555, Chem Phys. 27 (1978) 301.

[7] E.J. Heller, J Chem Phys 62 (1975) 1544, Chem Phys Letters 34 (1975) 321.

[8] R S Varga, Matrix iterative analysis (Prent ice-Hall, Englewood Cliffs, 1962)

[9] R C Tolman, The principles of statistical mechanics (Oxford Univ Press, London, 1938)

[10] H Lamb, Hydrodynamics, 6th Ed (Dover, New York, 1945)

[11] R L. Liboff, Introduction to the theory of kinetic equatrons (Wiley, New York, 1969)

[12] E K Grimmelmann and L L. Lohr Jr, to be published 